\title{
Mitochondrial DNA Alterations and Oxidative Stress in Acute Leukemia
}

\author{
Mouna Saadaoui ${ }^{1,4}$, Lamia Aissaoui ${ }^{2}$, Véronique Salaun ${ }^{3}$, Mohamed Manai ${ }^{4}$ and \\ Stéphane Allouche ${ }^{*}, 1$
}

\author{
${ }^{I}$ Université de Caen, Laboratoire de Biologie Moléculaire et Cellulaire de la Signalisation, UPRES-EA 3919, IFR 146 \\ ICORE, avenue côte de Nacre, 14032 Caen, France \\ ${ }^{2}$ Service d'Hématologie. CHU Aziza Othmana, Tunis, Tunisie \\ ${ }^{3}$ Service d'Hématologie Biologique, Avenue côte de Nacre, CHU de Caen, France \\ ${ }^{4}$ Unité de Biologie Moléculaire et de Biochimie, Faculté des Sciences de Tunis, Tunisie
}

\begin{abstract}
Mitochondrial DNA (mtDNA) alterations were reported in many cancers but their roles in oncogenesis are still debated. We aimed to examine qualitative and quantitative mtDNA modifications and oxidative stress in normal and cancer cells.

23 leukemia patients and 18 healthy subjects were recruited from the hospital of Azziza Othmana (Tunisia). Mitochondrial D-loop was sequenced, mtDNA level was determined by Q-PCR, the oxidative stress was assessed by a fluorescent probe and the mitochondrial transcription factor A (mTFA) level was quantified.

No somatic mutation was evidenced in leukemia cells compared to non-malignant cells. However, a significant higher level of mtDNA associated with an increase of mTFA expression and reactive oxygen species (ROS) production were measured in patients' cells compared to non-malignant cells.

In conclusion, our results don't support the role of mtDNA mutations in leukemogenesis but increase of mtDNA and ROS levels would be molecular signatures of leukemia.
\end{abstract}

Keywords: Mitochondrial DNA, leukemia, reactive oxygen species (ROS), mitochondrial transcription factor A (mTFA).

\section{INTRODUCTION}

Since the pioneering works of Otto Warburg in 1920s' about differences in the mitochondrial metabolism between normal and tumoral tissue, an abundant literature has been published to date pointing out alterations of mitochondria in cancer and their potential roles in carcinogenesis (see for review [1]). The human mtDNA contains $16.569 \mathrm{bp}$ [2] and plays an important role in energy metabolism. While the majority of mtDNA encodes ribosomal RNAs (rRNAs), transfer RNAs (tRNAs) and proteins, there is a small noncoding region of about $1.1 \mathrm{~kb}$ named D-loop which binds regulatory factors such as mitochondrial transcription factor A (mTFA) that plays a major role in the maintenance of mtDNA [3]. It's now well admitted that this region is a "hot spot" for mutations of mtDNA associated with various cancers [4] that would result from oxidative stress. Mitochondrion is the major site of reactive oxygen species (ROS) production. Under physiological conditions, ROS participate into various cell functions but their abnormal production or the loss of detoxifying enzyme would cause mtDNA damages and diseases [5].

*Address correspondence to this author at the Service de Biochimie, Centre Hospitalier et Universitaire, Avenue côte de nacre, 14033 Caen Cedex, France; Tel: +33 231065419; Fax: +33231065172;

E-mail: allouche-s@chu-caen.fr
Mitochondria were shown to be altered in hematological disorders. For instance, myelodysplastic syndromes are characterized by an ineffective hematopoiesis caused by mtDNA mutations that would directly be responsible for carcinogenesis [6]. Several laboratories also reported the presence of mtDNA mutations in chronic lymphocytic (CLL) and myeloid (CML) leukemia and in acute lymphoblastic (ALL) and myeloid (AML) leukemia [7-9]. However, a recent study challenged the potential role of mtDNA mutations in leukemogenesis [10].

Since the role of mitochondria in cancer is still a matter of debate, our study aimed to evaluate mtDNA instability within the D-loop and mtDNA copy number in Tunisian patients affected by acute leukemia. This was accomplished by $\mathrm{PCR} /$ sequencing and quantitative PCR of samples obtained from patients and healthy subjects. The significant increase in mtDNA content observed in tumor cells prompted us to examine mTFA and ROS, two factors known to regulate mitochondrial DNA replication. mTFA was quantified by real-time-RT-PCR and western-blot and ROS production was evaluated by hydroethidine/flow cytometry.

\section{MATERIALS AND METHODOLOGY}

\section{Patients}

Twenty three Tunisian children or young adults, with a mean age of 8.8 years and a sex ratio male/female of 1.87 , 
affected either by ALL $(n=16)$ or AML $(n=7)$ were studied (Table 1) and compared to eighteen healthy children or young adults from Tunisia with a sex ratio male/female of 1.25 and a mean age of 12.3 years. All patients, except P5, were included without prior chemotherapy. The study was conducted according to the Tunisian biomedical research rules that was approved by the ethics committee of the hospital of Azziza Othmana (Tunisia) and according to the ethical standards formulated in the Helsinki Declaration. All biological samples were collected anonymously.

Table 1. Characteristics of Patients Affected by Leukemia

\begin{tabular}{|c|c|c|c|c|c|}
\hline Samples & Sex & Age (Years) & Diagnosis & Evolution & Haplogroupe \\
\hline \hline P1 & M & 13 & ALL & CR & H \\
\hline P2 & M & 10 & ALL & ABMT & H \\
\hline P3 & M & 10 & ALL & CR & H \\
\hline P4 & M & 12 & AML & CR & L3f3 \\
\hline P5 & F & 3 & ALL & CR & L3f* \\
\hline P6 & F & 7 & ALL & CR & H \\
\hline P7 & F & 5 & ALL & CR & K \\
\hline P8 & F & 4 & ALL & Death & H11 \\
\hline P9 & F & 8 & AML & Death & H \\
\hline P10 & M & 4 & ALL & CR & J1b \\
\hline P11 & F & 10 & AML & ABMT & H5 \\
\hline P12 & F & 2 & ALL & CR & H \\
\hline P13 & F & 15 & AML & Death & H \\
\hline P14 & M & 20 & AML & Death & H \\
\hline P15 & M & 13 & ALL & CR & HV4a \\
\hline P16 & M & 9 & ALL & CR & H11 \\
\hline P17 & M & 7 & AML & ABMT & H \\
\hline P18 & M & 7 & ALL & CR & H \\
\hline P19 & M & 6 & ALL & CR & H \\
\hline P20 & M & 11 & AML & RE & H \\
\hline P21 & M & 17 & ALL & CR & H \\
\hline P22 & M & 3 & ALL & CR & H1f \\
\hline P23 & M & 8 & ALL & RE & H \\
\hline
\end{tabular}

M: male; F: female; CR: complete remission; RE: Relapse; ABMT: allogenic bone marrow transplantation; ALL: acute lymphoblastic leukemia; AML: acute myeloid leukemia.

\section{Peripheral Blood Mononuclear Cells Preparation}

Peripheral blood mononuclear cells (PBMC) were obtained from patients and healthy donors using a gradient Ficoll-Paque (Amersham Biosciences, Saclay France) and then were frozen at $-192^{\circ} \mathrm{C}$ into two vials for nucleic acid and protein extraction and measurement of superoxide anion $\left(\mathrm{O}_{2}{ }^{\circ}{ }^{\circ}\right)$ production.

\section{Nucleic Acid Extraction}

Total DNA was extracted from isolated PBMC and buccal cells by using the standard phenol/chloroform extraction procedure. Total RNAs were extracted from frozen PBMC cells of patients and healthy donors with TRIzol ${ }^{\circledR}$ reagent (Invitrogen, Saint-Aubin France). $3 \mu \mathrm{g}$ of total RNAs were reverse transcribed using the Moloney Murine Leukemia Virus Reverse Transcriptase (Invitrogen) to obtain cDNAs.

\section{mtDNA Sequencing}

Detection of polymorphisms/mutations in the D-loop of the mtDNA was performed by PCR using four primer pairs L1R1, L2R2, L3R3 and L4R4 (SUPPLEMENTARY MATERIAL 1) followed by direct sequencing using the Genomelab DTCS (Beckman Coulter, France) and analyzed on a Beckman Coulter CEQ 8000 sequencer. Estimation of mitochondrial haplogroups was determined using the mtDNAmanager interface developed by Hwan Young Lee et al. (2008) using the control region sequence [11].

\section{Determination of the mtDNA/nDNA Ratio by Real-Time PCR}

Quantification of the mtDNA was performed by real-time PCR amplification on a Light Cycler 480 (Roche Diagnostics, Meylan France) using Cycler FastStart DNA master Sybr green I mix (Roche Diagnostics) according to the method developed by May-Panloup et al. [12] except that we amplified the $18 \mathrm{~S}$ rRNA for nDNA quantification (SUPPLEMENTARY MATERIAL 1). All samples were analyzed in duplicate. Considering that 1 cell contains $10 \mathrm{pg}$ of nDNA, results are expressed as mtDNA copy number per cell.

\section{Real-Time PCR}

Real-time quantification of mTFA expression relative to $\beta$-actin mRNAs was performed on the Light Cycler 480 system (Roche Diagnostics) using specific set of primers (SUPPLEMENTARY MATERIAL 1). The threshold cycle $(\mathrm{Ct})$ was determined and the mTFA $/ \beta$-actin ratio was calculated according to the $2^{-\Delta \Delta C t}$ method [13].

\section{Western-Blot Analysis}

mTFA and $\beta$-actin expression were studied from PBMC by western-blot with a 1:500 dilution of rabbit anti-human mTFA (Abcam, Paris France) and a 1:1.000 dilution of the monoclonal anti- $\beta$-actin (santa-Cruz, Le Perray en Yvelines France) antibodies, respectively. Protein concentration was determined by the BCA Protein assay (Bicinchoninic Acid).

\section{Measurement of Superoxide Anion Production}

Superoxide anion radical production was determined by using the fluorescent sensitive probe hydroethidine (HE) (Molecular Probes, Invitrogen, Saint-Aubin France). PBMC $\left(5.10^{4}\right.$ cells $)$ obtained from controls or patients were incubated with $78 \mu \mathrm{M}$ HE for $15 \mathrm{~min}$ in PBS at $37^{\circ} \mathrm{C}$ in the dark. Then, fluorescence of oxidized HE was determined by flow cytometry (Becton Dickinson FACS CANTO II, FACS Diva Software version 6.1) with 485 and $510 \mathrm{~nm}$ wavelengths for excitation and emission, respectively.

\section{Statistical Analysis}

Data presented correspond to mean +/- S.E.M. The Fisher's exact test and the t-test were used to determine the statistical significance of differences between controls and 
patients. Differences were considered significant when $\mathrm{p}$ values were less than 0.05 . For multiple comparison tests, we applied the Bonferroni correction.

\section{RESULTS}

\section{mtDNA Sequence Analysis}

The D-loop region of the mtDNA was entirely sequenced in leukemia patients and healthy subjects. We observed several homoplasmic and heteroplasmic variations both in patients and controls compared to the rCRS (revised Cambridge Reference Sequence) (SUPPLEMENTARY MATERIAL 2A) but only the heteroplasmic m.343C $>$ T substitution was found significantly under-represented in patients compared to controls $(22 \%$ vs $89 \%$, p $<0.001$ respectively) (SUPPLEMENTARY MATERIAL 2B). In order to distinguish between somatic and germinal mutation/polymorphism, the $\mathrm{m} \cdot 343 \mathrm{C}>\mathrm{T}$ substitution was screened in buccal epithelial cells from 11 among 23 patients; an identical distribution of this polymorphism was found in both tissues.

\section{Quantitative Real Time PCR Analysis}

As the D-loop plays a major role in the regulation of mtDNA replication, quantification of mtDNA level was achieved from PBMC of patients and healthy donors by quantitative PCR using both mitochondrial and nuclear standards. As depicted in the Fig. (1A), a significant 3-fold increase in the mtDNA/nDNA ratio was measured between leukemia patients and controls (t-test, $p=0.012$ ). Values of mtDNA copy number from the control group were in the same range as previously reported in healthy donors with a different method and targed genes [14].

\section{mTFA Expression}

mTFA was described as a major activator of mtDNA transcription and replication. So, we sought to study the expression of this regulatory factor by real-time PCR and western-blot. We observed a significant increase in mTFA mRNAs in leukemia patients compared to controls ( $t$-test, $p$ $=0.009$ ) (Fig. 1B), that was correlated with the increase at the protein level (t-test, p <0.001) (Fig. 1C, D). The specificity of anti-mTFA antibody was verified using the Ramos and Jurkat cells lines in which we were able to detect a single band at the predicted size (data not shown).

\section{Measurement of ROS Production}

We measured the superoxide anion production in PBMC isolated from 14/23 leukemia patients and 11/18 healthy donors using the fluorescent probe HE. The more ROS are produced, the more fluorescence is increased (SUPPLEMENTARY MATERIAL 3). As shown in the Fig. (2), the level of ROS production, reflected by the mean fluorescence intensity, was significantly increased by about 2-fold in leukemia patients compared to controls (t-test, $\mathrm{p}=0.04$ ).
A

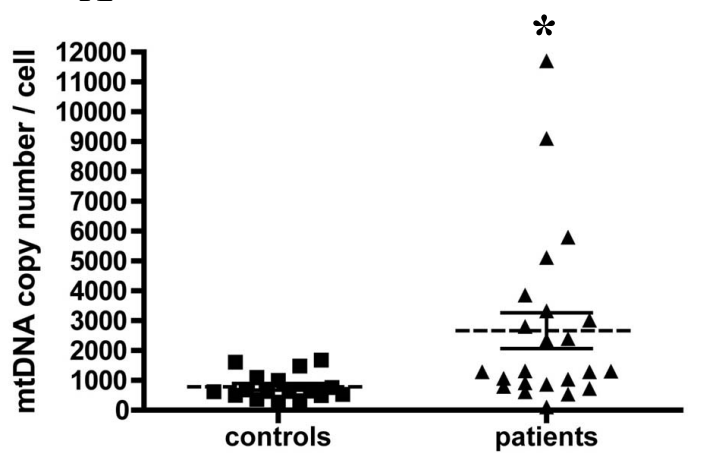

C

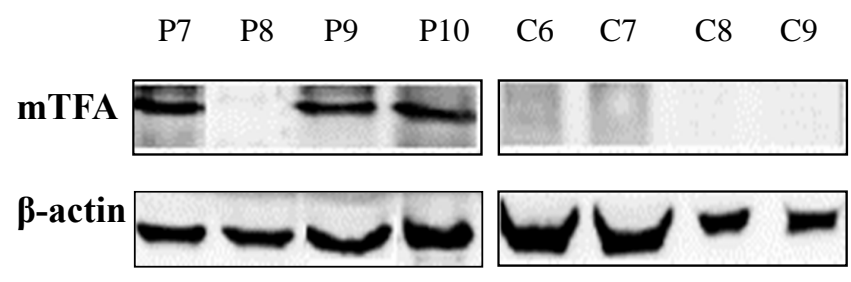

B

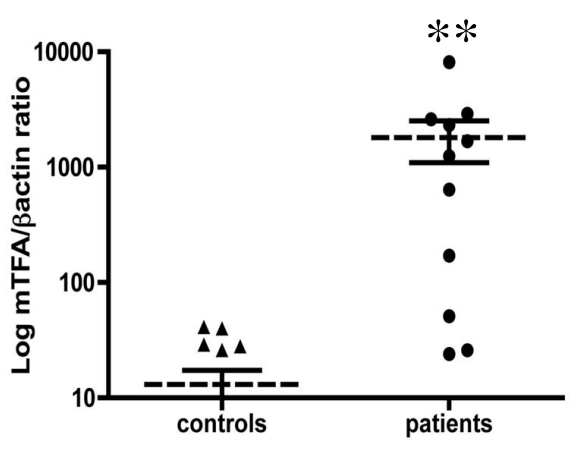

D

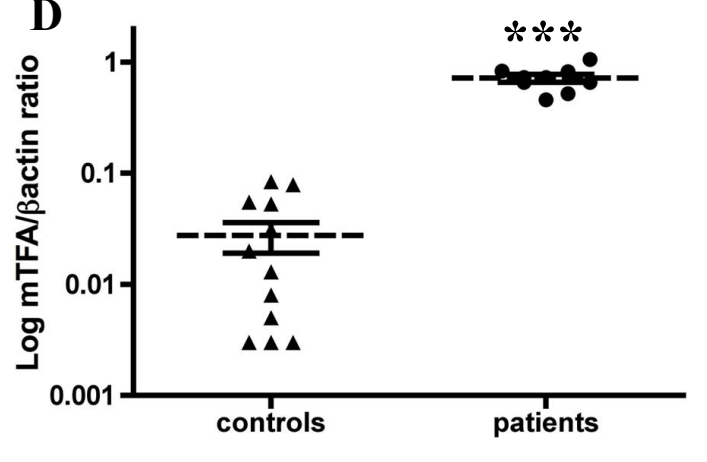

Fig. (1). Analysis of mtDNA and mTFA in leukemic patients and healthy subjects. (A) The mtDNA/nDNA ratio was determined by realtime PCR using primers directed against both mitochondrial and nuclear genes. Data are mean $+/-$ S.E.M. * P $<0.05$. (B) mRNAs expression of mTFA was determined by Q-PCR in 11/23 leukemic patients and 9/18 healthy controls. Results are expressed as the mTFA/ $\beta$ actin ratio and correspond to the mean + /- S.E.M. ${ }^{*}, \mathrm{P}<0.01$. (C, D) The mTFA level was determined by western-blot in $9 / 23$ leukemic patients and $13 / 18$ healthy controls. Results are expressed as the mTFA $/ \beta$ actin ratio and correspond to the mean $+/-$ S.E.M. $* * *, P<0.001$. 


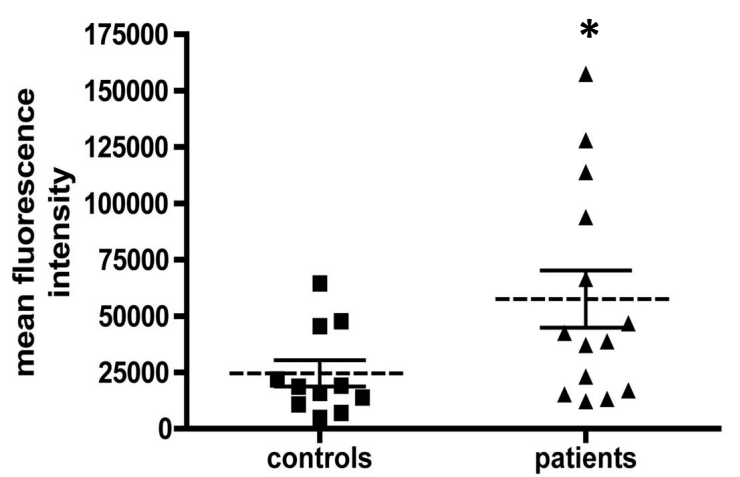

Fig. (2). Determination of ROS production in PBMC. ROS production was quantified using the fluorescent probe HE in cells extracted from leukemia patients and controls. ROS levels are reflected by the the mean fluorescence intensity. Data are mean $+/-$ S.E.M. *, $\mathrm{P}<0.05$.

\section{DISCUSSION}

There is an abundant literature about mtDNA mutations and cancers, both in solid tumors and leukemia but the relevance of such mitochondrial abnormalities in tumor genesis is still unclear (see for review [15]). These mtDNA point mutations are somatic and detected mainly in the Dloop. Regarding leukemia, mitochondrial mutations were also reported in this region and were suggested to contribute to tumor growth [8]. In contrast, our data don't support the existence of any link between mtDNA mutations and acute leukemia. Such conclusions have also been recently drawn by Cerezo et al. (2009) [10] from their study on B-cell CLL. While we detected a single significant difference at the position 343 between patients and healthy controls in PBMC, the epithelial buccal cells of patients, a nonmalignant tissue, also displayed the same $\mathrm{C}$ nucleotide in the homoplasmic state; this indicates that this mutation was not segregated in tumoral cells. There are different hypothesis to explain discrepancies between our data and those previously published:

- Our patients, except $\mathrm{N}^{\circ} 5$, didn’t receive neither chemotherapy nor platelets transfusion at the time of diagnosis that could lead to a false positive detection of polymorphism/mutation [16].

- $\quad$ D-loop is known to be a very polymorphic region and when using the rCRS as template, polymorphisms due to haplogroup can be mistaken as mutations. To avoid such pitfall and on the contrary of some studies [17] we rather compared D-loop sequences obtained from patients with controls coming from the same geographical region.

- We also checked that our PCR conditions did not cause amplification of nuclear pseudogenes closely related to mtDNA by using rho $^{\circ}$ cells.

- In our study, we compared tumoral (blood) and nonmalignant tissues (epithelial buccal cells) from same patients to determine the role of mtDNA mutation in cancer in contrast to some in which only the tumoral cells were studied [18].
We found that leukemia cells displayed a higher level of ROS production compared to non-malignant PBMC as recently reported in CML [19]. Indeed, alteration of the mitochondrial membrane potential was suggested to disturb electron flow through the mitochondrial respiratory chain and generate elevated level of ROS. While in our study we sequenced only the D-loop region, we can't rule out the presence of mutations or polymorphisms that would enhance the electron leakage and ROS production. Our data are also in good agreement with the study of Er et al. (2007) [20] who demonstrated higher superoxide anion production in AML compared to healthy controls. In such conditions, we would have expected to detect common oxidative damage signature mutations (C-T, CC-TT and G-T) of DNA in our patients as reported by Grist et al. (2004) [8]. However, in this study the authors selected a high proportion of patients who had been previously treated and relapsed; furthermore, data of mtDNA sequence analysis were compared to the rCRS. So, the mutations that were reported could be due to chemotherapy treatment or would correspond to polymorphisms. In addition to promote damages to cellular components and DNA mutations, ROS were suggested to play a role in intracellular signaling and to activate proproliferative and/ or survival pathways (see for review [21]). ROS would be deleterious in two ways: they would promote DNA instability by promoting DNA breaks and would confer a proliferative advantage in cancer cells. Due to the difficulty to maintain patients' cells in culture, we didn't test the role of ROS in proliferation.

ROS were also suggested to regulate the mtDNA copy number as recently demonstrated in nondiabetic hemodialysis patients [22]. In this study, the authors showed a good and positive correlation between mtDNA content in peripheral blood leukocytes and the plasma thiobarbituric acid-reactive substances level used as an indicator of oxidative stress. In the present study, we observed a significant increase in the $\mathrm{mtDNA} / \mathrm{nDNA}$ ratio in patients compared to controls. Such an increase was previously reported in blast cells of AML as well as during transformation of chronic granulocytic leukemia [23]. Aneuploidy is frequently observed in cancers including leukemia cells and could potentialy lead to erroneous nDNA quantification. However this is unlikely in our study since we used the $18 \mathrm{~S}$ rRNA which is a multicopy gene. mTFA, whose level is known to regulate the mtDNA copy number (see for review [3]), was increased in patients compared to healthy controls both at the levels of mRNA and protein. Very few data are available about the expression level of mTFA so it's difficult to compare our data. However, using a non-quantitative RT-PCR mTFA expression was shown to be absent in normal B-lymphocytes from 2 healthy donors while it was greatly expressed in CLL [24]. So it's not surprising to observe a strong increase in mTFA expression in patients compared to controls. Moreover, our molecular studies are in good agreement with western-blot experiments since in the control group mTFA was barely detectable while a strong immunoreactivity was measured in patients. We can question about signals that would trigger mTFA overexpression which consequently would induce increase in mtDNA level in leukemia patients. We can speculate that oxidative stress would damage mtDNA and compensatory mechanisms would be initiated to replace altered DNA 
molecules by new ones via mTFA. Such relationship between ROS and mTFA expression was previously reported in yeast mitochondria subjected to oxidative stress [25]. More, in addition to its role in mtDNA maintenance, mTFA was recently demonstrated to be localized in nucleus and its over-expression, in the human prostate cancer cell line PC3, was shown to induce cellular proliferation [26].

In conclusion, while our data suggest no evidence for mtDNA instability in leukemogenesis, we observed an increase in oxidative stress, mtDNA and mTFA levels in leukemia patients' cells. Such mitochondrial alterations could be used a molecular signature of leukemia cells. Further studies would be necessary to determine any link between such abnormalities and cell proliferation.

\section{ABBREVIATIONS}

$\begin{array}{lll}\text { ROS } & =\text { Reactive oxygen species } \\ \text { mTFA } & =\text { Mitochondrial transcription factor A } \\ \text { CLL } & =\text { Chronic lymphocytic leukemia } \\ \mathrm{CML} & =\text { Chronic myeloid leukemia } \\ \mathrm{ALL} & =\text { Acute lymphoblastic leukemia } \\ \mathrm{AML} & =\text { Acute myeloid leukemia } \\ \text { mtDNA } & =\text { Mitochondrial DNA } \\ \text { PBMC } & =\text { Peripheral blood mononuclear cells. }\end{array}$

\section{CONFLICT OF INTEREST}

The authors confirm that this article content has no conflict of interest.

\section{ACKNOWLEDGEMENTS}

Fundings come from the Ministères de l'enseignement supérieur et de la recherche Français et Tunisien. Mrs Mouna Saadaoui was supported by le Ministère de l'Enseignement Supérieur et de la Recherche Scientifique de Tunisie. We thank Dr. Balkis Meddeb for providing blood samples of the patients from Aziza Othmana Hospital, Dr. Inès Safra (laboratoire d'hématologie, Institut Pasteur de Tunis, Tunisie) for their advices, Dr. Christian Creveuil (Unité de biostatistique et de recherche clinique, CHU de Caen, France) for its statistical advices, Pr. Xavier Troussard (laboratoire d'hématologie, CHU de Caen, France) for his scientific collaboration and Dr. Florence Truquet (laboratoire d'hématologie, CHU de Caen, France) for her critical reading of the manuscript.

\section{FUNDING SOURCE}

Les ministères de l'enseignement supérieur et de la recherche Français et Tunisien.

\section{SUPPLEMENTARY MATERIAL}

Supplementary material is available on the publisher's web site along with the published article.

\section{REFERENCES}

[1] Koppenol WH, Bounds PL, Dang CV. Otto Warburg's contributions to current concepts of cancer metabolism. 2011; 11(5): 325-37.

[2] Anderson S, Bankier AT, Barrell BG, et al. Sequence and organization of the human mitochondrial genome. Nature 1981; 290: 457-65.

[3] Kang D, Kim SH, Hamasaki N. Mitochondrial transcription factor A (TFAM): roles in maintenance of mtDNA and cellular functions. Mitochondrion 2007; 7: 39-44.

[4] Chatterjee A, Dasgupta S, Sidransky D. Mitochondrial subversion in cancer. Cancer Prev Res (Phila) 2011; 4: 638-54.

[5] Fogg VC, Lanning NJ, Mackeigan JP. Mitochondria in cancer: at the crossroads of life and death. Chin J Cancer 2011; 30: 526-39.

[6] Wulfert M, Küpper AC, Tapprich C, et al. Analysis of mitochondrial DNA in 104 patients with myelodysplastic syndromes. Exp Hematol 2008; 36: 577-86.

[7] Carew JS, Zhou Y, Albitar M, Carew JD, Keating MJ, Huang P. Mitochondrial DNA mutations in primary leukemia cells after chemotherapy: clinical significance and therapeutic implications. Leukemia 2003;17:1437-47.

[8] Grist SA, Lu X-J, Morley AA. Mitochondrial mutations in acute leukaemia. Leukemia 2004;18:1313-6.

[9] He L, Luo L, Proctor SJ, et al. Somatic mitochondrial DNA mutations in adult-onset leukaemia. Leukemia 2003;17: 2487-91.

[10] Cerezo M, Bandelt H-J, Martín-Guerrero I, et al. High mitochondrial DNA Stability in B-Cell chronic lymphocytic leukemia. PLoS ONE 2009; 4: e7902.

[11] Lee HY, Song I, Ha E, Cho S-B, Yang WI, Shin K-J. mtDNAmanager: a Web-based tool for the management and quality analysis of mitochondrial DNA control-region sequences. BMC Bioinformatics 2008; 9: 483.

[12] May-Panloup P, Chrétien M-F, Savagner F, et al. Increased sperm mitochondrial DNA content in male infertility. Hum Reprod 2003; 18: 550-6.

[13] Livak KJ, Schmittgen TD. Analysis of relative gene expression data using real-time quantitative PCR and the 2(-Delta Delta C(T)) Method. Methods 2001; 25: 402-8.

[14] Gourlain K, Amellal B, Ait Arkoub Z, Dupin N, Katlama C, Calvez V. Quantitative analysis of human mitochondrial DNA using a realtime PCR assay. HIV Med 2003; 4: 287-92.

[15] Lu J, Sharma LK, Bai Y. Implications of mitochondrial DNA mutations and mitochondrial dysfunction in tumorigenesis. Cell Res 2009; 19: 802-15.

[16] Meierhofer D, Ebner S, Mayr JA, Jones ND, Kofler B, Sperl W. Platelet transfusion can mimic somatic mtDNA mutations. Leukemia 2006; 20: 362-3.

[17] Sharawat SK, Bakhshi R, Vishnubhatla S, Bakhshi S. Mitochondrial D-loop variations in paediatric acute myeloid leukaemia: a potential prognostic marker. Br J Haematol 2010; 149: 391-8.

[18] Kwok CS-N, Quah TC, Ariffin H, Tay SK-H, Yeoh AE-J. Mitochondrial D-loop polymorphisms and mitochondrial DNA content in childhood acute lymphoblastic leukemia. J Pediatr Hematol Oncol 2011; 33: e239-44.

[19] Nieborowska-Skorska M, Kopinski PK, Ray R, et al. Rac2mitochondrial respiratory chain complex III-generated ROS cause genomic instability in chronic myeloid leukemia stem cells and primitive progenitors. Blood 2012; 119(18): 4253-63.

[20] Er T-K, Tsai S-M, Wu S-H, et al. Antioxidant status and superoxide anion radical generation in acute myeloid leukemia. Clin Biochem 2007; 40: 1015-9.

[21] Hole PS, Darley RL, Tonks A. Do reactive oxygen species play a role in myeloid leukemias? Blood 2011;117: 5816-26.

[22] Wang Y-C, Lee W-C, Liao S-C, et al. Mitochondrial DNA copy number correlates with oxidative stress and predicts mortality in nondiabetic hemodialysis patients. J Nephrol 2011; 24: 351-8.

[23] Boultwood J, Fidler C, Mills KI, et al. Amplification of mitochondrial DNA in acute myeloid leukaemia. Br J Haematol 1996; 95: 426-31. 
[24] Carew JS, Nawrocki ST, Xu RH, et al. Increased mitochondrial biogenesis in primary leukemia cells: the role of endogenous nitric oxide and impact on sensitivity to fludarabine. Leukemia 2004; 18 : 1934-40.

[25] Hori A, Yoshida M, Shibata T, Ling F. Reactive oxygen species regulate DNA copy number in isolated yeast mitochondria by triggering recombination-mediated replication. Nucleic Acids Res 2009; 37: 749-61.

[26] Han B, Izumi $\mathrm{H}$, Yasuniwa $\mathrm{Y}$, et al. Human mitochondrial transcription factor $\mathrm{A}$ functions in both nuclei and mitochondria and regulates cancer cell growth. Biochem Biophys Res Commun 2011; 408: 45-51.

(C) Saadaoui et al.; Licensee Bentham Open.

This is an open access article licensed under the terms of the Creative Commons Attribution Non-Commercial License (http: //creativecommons.org/licenses/by$\mathrm{nc} / 3.0 /$ ) which permits unrestricted, non-commercial use, distribution and reproduction in any medium, provided the work is properly cited. 